\title{
The importance of non-tuberculous mycobacteria identification in Chinese patients infected with HIV
}

\author{
Li Liu ${ }^{1}$, Renfang Zhang ${ }^{1}$, Yang Tang ${ }^{1}$, Tangkai Qi ${ }^{1}$, Wei Song ${ }^{1}$, Zhenyan Wang ${ }^{1}$, Yinzhong Shen ${ }^{1}$, \\ Hongzhou $\mathrm{Lu}^{1,2, *}$
}

${ }^{1}$ Department of Infectious Disease, Shanghai Public Health Clinical Center, Fudan University, Shanghai, China;

${ }^{2}$ Department of Infectious Disease, Huashan Hospital Affiliated to Fudan University, Shanghai, China.

\begin{abstract}
Summary The increased co-incidence of tuberculosis (TB) and AIDS is compounded by the emergence of opportunistic infections with non-tuberculous mycobacteria (NTM) in patients with HIV/ AIDS, and the treatment for these infections differs from that for TB. The high frequency of NTM strains found in patients infected with HIV raises concerns about accurate species identification before deciding proper treatment. A total of 101 isolates from 2014, 137 from 2015, and 162 from 2016 were subjected to $16 \mathrm{~S}$ rDNA sequencing to identify the species. Forty-one (41/101, 40.6\%) were identified as NTM in 2014, 64 (64/137, 46.7\%) were identified as NTM in 2015, and $72(72 / 162,44.4 \%)$ were identified as NTM in 2016 in Chinese patients infected with HIV. The species of Mycobacteria isolates needs to be rapidly and accurately identified to determine appropriate antibiotic therapy, and this is especially true for patients infected with HIV.
\end{abstract}

Keywords: Non-tuberculous mycobacteria, tuberculosis (TB), HIV, identification

People living with human immunodeficiency virus (HIV) are approximately 30 times more likely to develop tuberculosis (TB) than individuals without HIV. TB is the most common presenting illness among people living with HIV, including those receiving antiretroviral treatment, and the major cause of HIV-related death (1). The TB-HIV/AIDS epidemic is compounded by the emergence of opportunistic infections with nontuberculous mycobacteria (NTM) in patients with HIV/ AIDS patients, and the treatment for these infections differs from that for TB. In patients with AIDS, the clinical and radiological features of infections with NTM and tuberculous mycobacteria (MTB) are more complex and usually involve different disease patterns and atypical clinical findings, thus limiting the diagnosis of mycobacterial infections in patients infected with HIV (23). Given the high prevalence of HIV infection in China, there is a growing concern that NTM and other infections could be misdiagnosed as MTB in people infected with

\footnotetext{
*Address correspondence to:

Dr. Hongzhou Lu, Shanghai Public Health Clinical Center, Fudan University, No.2901, Caolang Road, Jinshan District, Shanghai 201508, China.

E-mail: luhongzhou@fudan.edu.cn
}

HIV. A recent report from Guangxi, China indicated that M. tuberculosis was found in 117 patients infected with HIV (53\%) while NTM was found in 102 (47\%) (4). The current study has determined the frequency with which MTB and NTM strains were isolated from patients infected with HIV in Shanghai from 2014 to 2016 and the genotype of NTM in this population.

A total of 101 isolates from 2014, 137 from 2015, and 162 from 2016 were subjected to $16 \mathrm{~S}$ rDNA sequencing to identify the species. Forty-one $(41 / 101,40.6 \%)$ were identified as NTM in 2014, 64 (64/137, 46.7\%) were identified as NTM in 2015, and 72 (72/162, 44.4\%) were identified as NTM in 2016 (Figure 1). A total of 7 NTM species or complexes were identified from the NTM isolates (Table 1). The organism detected most frequently was M. avium complex (MAC), followed by M. gordonae and M. kansasii.

The high frequency of NTM strains found in patients infected with HIV raises concerns about accurate species identification before deciding proper treatment (5). Failure to characterize acid-fast bacilli (AFB)positive lung infections caused by NTM has led to the misdiagnosis of these infections and mistreatment for pulmonary TB in developing countries such as China. Treatment is limited because of the difficulty in 
Table 1. Identification of the species of NTM isolates

\begin{tabular}{lccc}
\hline Year & 2014 & 2015 & 2016 \\
\hline M. avium complex (MAC) & $17(17 / 41,41.5 \%)$ & $26(26 / 64,40.6 \%)$ & $32(32 / 72,44.4 \%)$ \\
M. gordonae & $10(10 / 41,24.3 \%)$ & $20(20 / 64,31.3 \%)$ & $19(19 / 72,26.4 \%)$ \\
M. kansasii & $11(11 / 41,26.8 \%)$ & $15(15 / 64,23.4 \%)$ & $18(18 / 72,25 \%)$ \\
M. fortuitum & $2(2 / 41,4.9 \%)$ & 0 & 0 \\
M. abscessus & 0 & 0 & $3(1 / 72,1.4 \%)$ \\
M. xenopi & 0 & 0 & $2(2 / 72,2.8 \%)$ \\
M. szulgai & $1(1 / 41,2.4 \%)$ & 0 & 0 \\
\hline
\end{tabular}

M: mycobacteria.

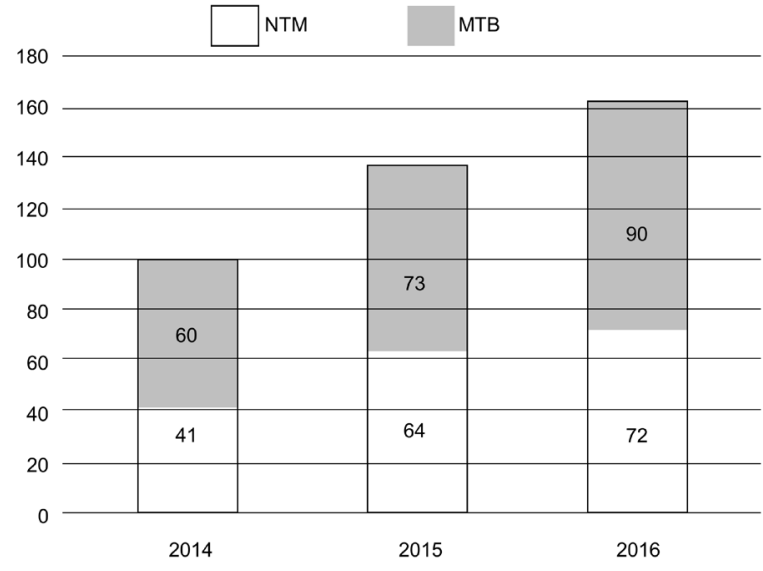

Figure 1. Mycobacterium tuberculosis and non-tuberculous mycobacteria isolates from patients infected with HIV from 2014-2016 in Shanghai, China. A total of 101,137 M. tuberculosis isolates and 162 non-tuberculous mycobacterium (NTM) isolates were subjected to $16 \mathrm{~S}$ rDNA sequencing to identify the species. Forty-one samples $(41 / 101,40.6 \%)$ were identified as NTM in 2014, $64(64 / 137,46.7 \%$ ) were identified as NTM in 2015 , and $72(72 / 162,44.4 \%)$ were identified as NTM in 2016.

identifying the species of pathogen up and the resistance of most strains of NTM to conventional anti-MTB drugs (6). When treated empirically, some NTM infections are misdiagnosed as non-responsive or drug-resistant MTB cases, and this can result in poor outcomes for those patients (7). Overall, the species of Mycobacteria isolates needs to be rapidly and accurately identified to determine appropriate antibiotic therapy, and this is especially true for patients infected with HIV (8). However, many clinical laboratories in designated HIV/AIDS care units do not have proper facilities and experienced technicians, thus hampering the accurate diagnosis of mycobacterial infections in HIV/AIDS patients in China. All positive mycobacterial isolates need to be sent to an authorized lab for further identification. Conventional biochemical tests are time-consuming and their results are not reproducible, while novel molecular biology techniques and high-performance liquid chromatographic analysis of mycolic acid can be performed accurately and rapidly.

\section{Acknowledgements}

This research was funded by a grant from the Shanghai Municipal Commission of Health and Family Planning (grant no. 15GWZK0103 ).

\section{References}

1. Abubakar I, Gupta RK1, Rangaka MX, Lipman M. Update in tuberculosis and nontuberculous mycobacteria 2017. Am J Respir Crit Care Med. 2018; 197:1248-1253.

2. Nyamogoba HD, Mbuthia G, Mining S, Kikuvi G, Biegon R, Mpoke S, Menya D, Waiyaki PG. HIV co-infection with tuberculous and non-tuberculous mycobacteria in western Kenya: Challenges in the diagnosis and management. Afr Health Sci. 2012; 12:305-311.

3. Brode S.K, Daley C.L, Marras T.K. The epidemiologic relationship between tuberculosis and nontuberculous mycobacterial disease: A systematic review. Int J Tuberc Lung Dis. 2014; 18:1370-1377

4. Lan R, Yang C, Lan L, Ou J, Qiao K, Liu F, Gao Q. Mycobacterium tuberculosis and non-tuberculous mycobacteria isolates from HIV-infected patients in Guangxi, China. Int J Tuberc Lung Dis. 2011; 15:16691675.

5. Henkle E, Winthrop KL. Nontuberculous mycobacteria infections in immunosuppressed hosts. Clin Chest Med. 2015; 36:91-99.

6. Hoza AS, Mfinanga SG, Rodloff AC, Moser I, König B. Increased isolation of nontuberculous mycobacteria among TB suspects in Northeastern, Tanzania: Public health and diagnostic implications for control programmes. BMC Res Notes. 2016; 9:109.

7. Bjerrum S, Oliver-Commey J, Kenu E, Lartey M, Newman MJ, Addo KK, Hilleman D, Andersen AB, Johansen IS. Tuberculosis and non-tuberculous mycobacteria among HIV-infected individuals in Ghana. Trop Med Int Health. 2016; 21:1181-1190.

8. Kobayashi T, Nishijima T, Teruya K, Aoki T, Kikuchi Y, Oka S, Gatanaga H. High mortality of disseminated non-tuberculous mycobacterial infection in HIV-infected patients in the antiretroviral therapy era. PLoS One. 2016; 11:e0151682.

(Received October 19, 2018; Accepted October 25, 2018) 